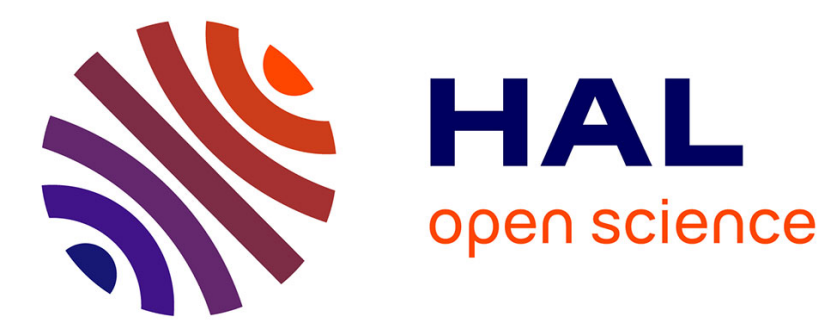

\title{
Collaborative and multilevel optimizations of a hybrid railway power substation
}

\author{
Stephane Brisset, Maxime Ogier
}

\section{To cite this version:}

Stephane Brisset, Maxime Ogier. Collaborative and multilevel optimizations of a hybrid railway power substation. International Journal of Numerical Modelling: Electronic Networks, Devices and Fields, 2017, pp.1-13. 10.1002/jnm.2289 . hal-01629326

\section{HAL Id: hal-01629326 \\ https://inria.hal.science/hal-01629326}

Submitted on 6 Nov 2018

HAL is a multi-disciplinary open access archive for the deposit and dissemination of scientific research documents, whether they are published or not. The documents may come from teaching and research institutions in France or abroad, or from public or private research centers.
L'archive ouverte pluridisciplinaire HAL, est destinée au dépôt et à la diffusion de documents scientifiques de niveau recherche, publiés ou non, émanant des établissements d'enseignement et de recherche français ou étrangers, des laboratoires publics ou privés. 


\title{
COLLABORATIVE AND MULTILEVEL OPTIMIZATIONS OF A HYBRID RAILWAY POWER SUBSTATION
}

\author{
Stephane BRISSET* and Maxime OGIER** \\ *Univ. Lille, Centrale Lille, Arts et Metiers ParisTech, HEI, EA 2697 - L2EP - Laboratoire \\ d'Electrotechnique et d'Electronique de Puissance, F-59000 Lille, France \\ E-mail: stephane.brisset@centralelille.fr \\ **Univ. Lille, CNRS, Centrale Lille, Inria, UMR 9189 - CRIStAL - Centre de Recherche en \\ Informatique Signal et Automatique de Lille, F-59000 Lille, France \\ E-mail: maxime.ogier@centralelille.fr
}

\begin{abstract}
Three approaches are proposed for the design of a hybrid railway power substation taking into account the control of the storage system over one year. The first one is based on a piecewise linearization of the cost function and solved with linear programming. The others decompose the whole problem in two levels with lower dimensionality. Collaborative optimization solves it with a double-loop scheme while Benders decomposition allows a sequential resolution. This last gives the same solution as linear programming in about 5 times more computing time and can be used with a non-linear cost function.
\end{abstract}

Keywords: Hybrid railway power substation, multi-source systems, energy storage, collaborative optimization, Benders decomposition.

\section{INTRODUCTION}

Because of the rail network development, power demand of railway power substation (RPS) becomes increasingly important [1]. The integration of renewable energies could compensate this need and it reduces pollution. Nevertheless the known intermittency factor of these energies makes it difficult to estimate the amount of produced power [2]. By introducing a storage device, the hybrid RPS (HRPS) could ensure a good power quality and it reduces total cost [1]. The storage device should be accurately used according to consumed power, produced power and evolution of prices fixed by the power supplier [3]. To enhance the design of HRPS, energy management strategy should be suitable in the long term. This requirement leads to a very high number of decision variables that is 52560 for a time span of one year and measurement every ten minutes.

This work falls within an emerging and challenging topic in electric engineering where the main issue is to design complex systems and networks for which the dimensional and control parameters have to be optimized all at once because of their strong interaction [2]-[5]. Renewable energies like photovoltaic panels [2], wind turbines [3], and wave energy converters [5] are typically intermittent with variations during the day and the seasons. The use of storage systems can balance climatic irregularity but their control along a large operating profile, that is typically one year or more, introduces numerous parameters leading to large-scale optimization problems.

Three approaches could be used for the design by optimization of HRPS. The first one is to linearize the cost function and solve the energy formulation problem by using linear programming (LP) in order to find an approximate solution. If the problem scale is high and the functions are non-linear then decomposition methods can be applied to divide the complete problem into sub-problems with lower dimensionality. In this paper, collaborative optimization (CO) and Benders decomposition (BD) are investigated to find an exact solution of the whole problem. However, the difficulty is in the coordination of sub-optimizations and the convergence to the solution of the whole problem.

In the first and second parts, the model and the optimization problem of HRPS are presented. The third part details the assumptions made to linearize the problem and compares the memory required by power and energy formulations with linear programming. Collaborative and multilevel optimizations are explained and applied to the HRPS problem in parts four and five, respectively. The three approaches are compared in terms of precision and computing time in part six. Finally, some conclusions and prospects are given.

\section{MODEL OF HYBRID RAILWAY POWER SUBSTATION}

The studied HRPS is presented in Fig. 1, it is composed of photovoltaic panels, wind turbines and a storage system connected to the substation through an AC bus. Renewable energy productions are illustrated by one-way power flows on Fig. 1. 


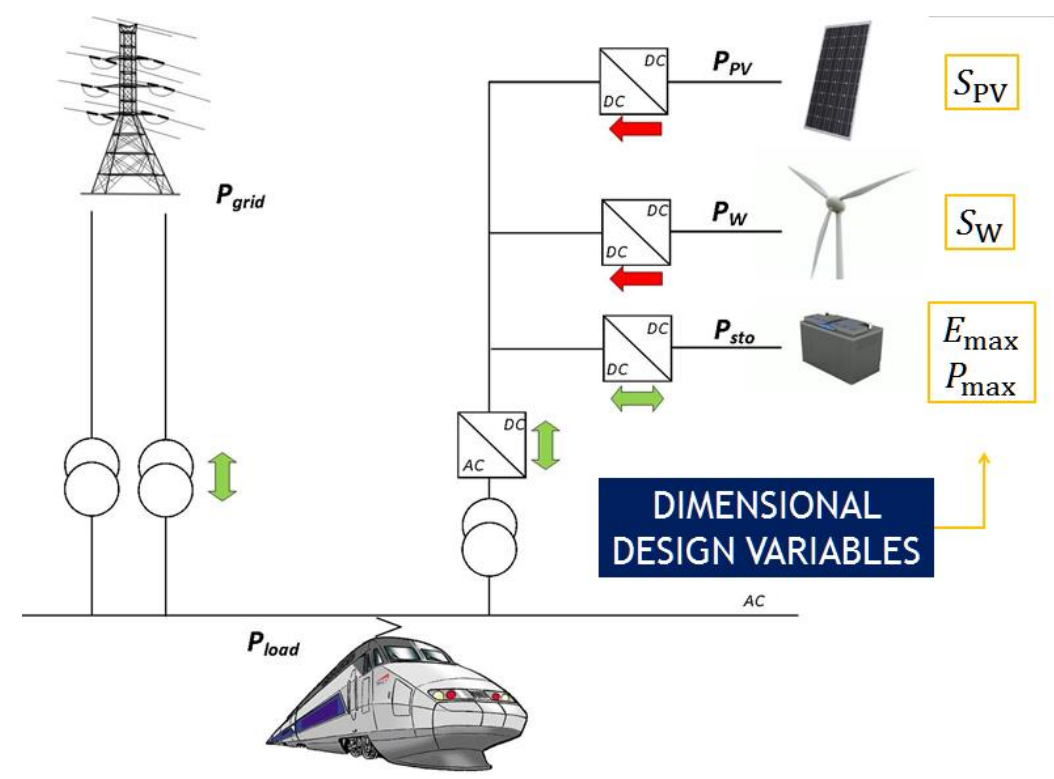

Figure 1. Power flows in hybrid railway power substation and dimensional decision variables.

Their powers vary over time and depend on the photovoltaic panels surface $S_{\mathrm{PV}}$, and the swept surface of wind turbines $S_{\mathrm{W}}$ :

$$
\begin{aligned}
& P_{\mathrm{PV}}(t)=S_{\mathrm{PV}} P_{\mathrm{irr}}(t) \quad \forall t \in[0, T] \\
& P_{\mathrm{W}}(t)=k_{\mathrm{p}} S_{\mathrm{W}} v_{\text {adj }}^{3}(t) \quad \text { with } \quad v_{\text {adj }}(t)=\left\{\begin{array}{cc}
v_{\mathrm{W}}(t) & 0 \leq v_{\mathrm{W}}(t)<v_{\text {rated }} \\
v_{\text {rated }} & v_{\text {rated }} \leq v_{\mathrm{W}}(t) \leq v_{\text {off }} \\
0 & v_{\text {off }}<v_{\mathrm{W}}(t)
\end{array} \quad \forall t \in[0, T]\right.
\end{aligned}
$$

where $T$ is the end of time range, $v_{\mathrm{W}}(t)$ is the wind speed in $\mathrm{m} / \mathrm{s}, P_{\mathrm{irr}}(t)$ is the solar irradiance in $\mathrm{W} / \mathrm{m}^{2}, v_{\text {off }}$ is the turbine cut-off wind speed in $\mathrm{m} / \mathrm{s}, v_{\text {rated }}$ is the turbine rated wind speed in $\mathrm{m} / \mathrm{s}$, and $k_{\mathrm{p}}=\rho_{\text {air }} C_{p} / 2$ where $\rho_{\text {air }}$ is the air density and $C_{p}$ the power coefficient of the wind turbine, assumed constant.

All power flows of this multi-source system have to satisfy the power balance:

$$
P_{\text {load }}(t)-P_{\text {sto }}(t)-P_{\mathrm{PV}}(t)-P_{\mathrm{W}}(t)-P_{\text {grid }}(t)=0 \quad \forall t \in[0, T]
$$

where $P_{\text {grid }}(t)$ is the power from the grid, $P_{\text {sto }}(t)$ is the power from the storage system, and $P_{\text {load }}(t)$ is the power consumption of trains. Given the maximum amount of stored energy and power exchanged between the storage system and other substation components, lower and upper bounds apply for $P_{\text {sto }}(t)$ and $E_{\text {sto }}(t)$ as expressed in (4)-(5):

$$
\begin{aligned}
& -P_{\max } \leq P_{\text {sto }}(t) \leq P_{\max } \quad \forall t \in[0, T] \\
& 0 \leq E_{\text {sto }}(t) \leq E_{\max } \quad \forall t \in[0, T]
\end{aligned}
$$

where the maximum amounts of power $P_{\max }$ in W and energy $E_{\max }$ in J define the storage capacity. At the end of the time range $T$, the amount of energy stored is supposed to return to its initial value $E_{\text {ini }}$ for periodicity:

$$
E_{\text {sto }}(T)=E_{\text {ini }}
$$

The power $P_{\text {sto }}(t)$ and the energy $E_{\text {sto }}(t)$ can both express the temporal fluctuation of energy in the storage device. The stored energy is computed from the history of $P_{\text {sto }}(t)$ and the initial amount of energy in the storage system $E_{\text {ini }}$ assuming that there is no loss in the storage system:

$$
E_{\text {sto }}(t)=E_{\text {ini }}-\int_{\tau=0}^{t} P_{\text {sto }}(\tau) d \tau \quad \forall t \in[0, T]
$$

Reciprocally, the power $P_{\text {sto }}(t)$ can be computed from the derivative of the stored energy $E_{\text {sto }}(t)$ : 


$$
P_{\text {sto }}(t)=-d E_{\text {sto }}(t) / d t \quad \forall t \in[0, T]
$$

\section{OPTIMIZATION PROBLEMS}

In equations (1)-(8), the values of the wind speed $v_{\mathrm{W}}(t)$, the solar irradiance $P_{\mathrm{irr}}(t)$, the turbine cut-off wind speed $v_{\text {off }}$, the turbine rated wind speed $v_{\text {rated }}$, and the power consumption of trains $P_{\text {load }}(t)$ are known. In order to solve the optimization problem, the time is discretized with a time step $\Delta t$ resulting in $n_{\mathrm{t}}=T / \Delta t$ time steps. Measurement of the mean power consumption of trains is made every 10 minutes therefore, the time step is taken equal to $\Delta t=600$ seconds. The wind speed and solar irradiance are measured every hour and interpolations are used to compute values every 600 seconds. The time range is considered to be one year as the slowest variation is the one of the solar irradiance, resulting in $n_{\mathrm{t}}=52560$ time steps.

Now, it is possible to write the discretized forms of equations (7) and (8):

$$
\begin{array}{ll}
E_{\text {sto }}(t=i \Delta t)=E_{\text {sto }, \mathrm{i}}=E_{\mathrm{ini}}-\Delta t \sum_{\mathrm{j}=1}^{\mathrm{i}} P_{\text {sto }, \mathrm{j}} & \forall i \in \llbracket 1, n_{\mathrm{t}} \rrbracket \\
P_{\text {sto }}(t=i \Delta t)=P_{\text {sto }, \mathrm{i}}=\left[E_{\mathrm{sto}, \mathrm{i}-1}-E_{\text {sto }, \mathrm{i}}\right] / \Delta t & \forall i \in \llbracket 1, n_{\mathrm{t}} \rrbracket \quad \text { with } \quad E_{\text {sto }, 0}=E_{\mathrm{ini}}
\end{array}
$$

The objective function is expressed in (9) for $n_{\mathrm{y}}=20$ years operating mode, that is the lifespan of equipments, by considering investments $\cos t C_{\mathrm{inv}}$ of all devices and also the non-linear energy cost for purchasing or selling electricity. Bold letters are used for vectors.

$$
\mathrm{OF}\left(S_{\mathrm{PV}}, S_{\mathrm{W}}, P_{\text {max }}, E_{\text {max }}, \boldsymbol{P}_{\text {grid }}\right)=C_{\mathrm{inv}}\left(S_{\mathrm{PV}}, S_{\mathrm{W}}, P_{\text {max }}, E_{\text {max }}\right)+n_{\mathrm{y}} \sum_{\mathrm{i}=1}^{\mathrm{n}_{\mathrm{t}}} \operatorname{cost}\left(P_{\text {grid }, \mathrm{i}}\right)
$$

The decision variables are the photovoltaic panels surface $S_{\mathrm{PV}}$, the swept surface of wind turbines $S_{\mathrm{W}}$, the maximum amount of power $P_{\max }$, the maximum amount of energy in the storage system $E_{\max }$, the initial amount of energy in the storage system $E_{\text {ini }}$, and a high dimension decision variable for the control of the storage system that can be the power $\boldsymbol{P}_{\text {sto }}$ or the energy $\boldsymbol{E}_{\text {sto }}$. Therefore, two optimization problems can be expressed.

In the first formulation, the decision variables for the control of the storage system are the powers $P_{\text {sto }}(t)$ and $(7 d)$ is used to rewrite the discretized forms of equations (4)-(6):

$$
\begin{aligned}
& -P_{\max } \leq P_{\text {sto }, \mathrm{i}} \leq P_{\max } \quad \forall i \in \llbracket 1, n_{\mathrm{t}} \rrbracket \\
& 0 \leq E_{\mathrm{ini}}-\Delta t \sum_{\mathrm{j}=1}^{\mathrm{i}} P_{\text {sto }, \mathrm{j}} \leq E_{\max } \quad \forall i \in \llbracket 1, n_{\mathrm{t}} \rrbracket \\
& \sum_{\mathrm{j}=1}^{\mathrm{n}_{\mathrm{t}}} P_{\text {sto }, \mathrm{j}}=0
\end{aligned}
$$

Equations (1)-(3) are merged to express the power from the grid:

$$
P_{\text {grid }, \mathrm{i}}=P_{\text {load }, \mathrm{i}}-P_{\text {sto }, \mathrm{i}}-S_{\mathrm{PV}} P_{\mathrm{irr}, \mathrm{i}}-k_{\mathrm{p}} S_{\mathrm{W}} v_{\mathrm{adj}, \mathrm{i}}^{3} \quad \forall i \in \llbracket 1, n_{\mathrm{t}} \rrbracket
$$

Therefore, the optimization problem with the power formulation is stated as:

$$
\begin{array}{r}
\min _{S_{\mathrm{PV}}, S_{\mathrm{W}}, P_{\mathrm{max}}, E_{\mathrm{max}}, E_{\mathrm{ini}}, \boldsymbol{P}_{\text {sto }}} \operatorname{OF}\left(\begin{array}{c}
S_{\mathrm{PV}}, S_{\mathrm{W}}, P_{\mathrm{max}}, E_{\mathrm{max}}, \\
\boldsymbol{P}_{\text {load }}-\boldsymbol{P}_{\text {sto }}-S_{\mathrm{PV}} \boldsymbol{P}_{\text {irr }}-k_{\mathrm{p}} S_{W} \boldsymbol{v}_{\mathrm{adj}}^{3}
\end{array}\right) \\
\text { s.t. }(4 \mathrm{~d}),(10),(11) \\
S_{\mathrm{PV}} \in R^{+}, S_{\mathrm{W}} \in R^{+}, P_{\max } \in R^{+}, E_{\mathrm{max}} \in R^{+}, E_{\mathrm{ini}} \in R^{+}, \boldsymbol{P}_{\text {sto }} \in R^{\mathrm{n}_{\mathrm{t}}}
\end{array}
$$

In the second formulation, the decision variables for the control of the storage system are the energies $E_{\text {sto }}(t)$ and (8) is used to rewrite equations (4)-(6) and (12):

$$
0 \leq E_{\text {sto }, \mathrm{i}} \leq E_{\max } \quad \forall i \in \llbracket 1, n_{\mathrm{t}} \rrbracket
$$

(6d) $\quad E_{\text {sto, } \mathrm{n}_{\mathrm{t}}}=E_{\text {ini }}$

$$
\begin{aligned}
& -P_{\max } \Delta t \leq E_{\mathrm{sto}, \mathrm{i}-1}-E_{\mathrm{sto}, \mathrm{i}} \leq P_{\mathrm{max}} \Delta t \quad \forall i \in \llbracket 1, n_{\mathrm{t}} \rrbracket \quad \text { with } \quad E_{\mathrm{sto}, 0}=E_{\mathrm{ini}} \\
& P_{\text {grid }, \mathrm{i}}=P_{\text {load }, \mathrm{i}}-\Delta E_{\mathrm{sto}, \mathrm{i}} / \Delta t-S_{\mathrm{PV}} P_{\mathrm{irr}, \mathrm{i}}-k_{\mathrm{p}} S_{\mathrm{W}} v_{\mathrm{adj}, \mathrm{i}}^{3} \quad \forall i \in \llbracket 1, n_{\mathrm{t}} \rrbracket \quad \text { with } \Delta E_{\text {sto }, \mathrm{i}}=E_{\mathrm{sto}, \mathrm{i}-1}-E_{\mathrm{sto}, \mathrm{i}}
\end{aligned}
$$


Therefore, the optimization problem with the energy formulation is stated as:

$$
\begin{gathered}
\min _{S_{\mathrm{PV}}, S_{\mathrm{W}}, P_{\mathrm{max}}, E_{\mathrm{max}}, E_{\mathrm{ini}}, E_{\mathrm{sto}}} \operatorname{OF}\left(\begin{array}{c}
S_{\mathrm{PV}}, S_{\mathrm{W}}, P_{\mathrm{max}}, E_{\mathrm{max}} \\
\boldsymbol{P}_{\text {load }}-\Delta \boldsymbol{E}_{\text {sto }} / \Delta t-S_{\mathrm{PV}} \boldsymbol{P}_{\mathrm{irr}}-k_{\mathrm{p}} S_{\mathrm{W}} \boldsymbol{v}_{\mathrm{adj}}^{3}
\end{array}\right) \\
\text { s.t. }(5 \mathrm{~d}),(6 \mathrm{~d}),(14) \\
S_{\mathrm{PV}} \in R^{+}, S_{\mathrm{W}} \in R^{+}, P_{\mathrm{max}} \in R^{+}, E_{\mathrm{max}} \in R^{+}, E_{\mathrm{ini}} \in R^{+}, \boldsymbol{E}_{\text {sto }} \in R^{+\mathrm{n}_{\mathrm{t}}}
\end{gathered}
$$

To summarize, the optimization problems for both formulations include $n_{\mathrm{t}}+5=52565$ design variables, one non-linear objective function, one linear equality constraint, and $4 n_{\mathrm{t}}=210240$ linear inequality constraints. It is to mention that the left inequality in $(5 \mathrm{~d})$ for the optimization problem with the energy formulation that is $0 \leq E_{\text {sto,i }}$ can be ignored since the lower bound of energy is zero. This removes $n_{\mathrm{t}}=52560$ inequality constraints.

\section{LINEARIZATION OF COSTS}

Several pricing systems exist in the electricity market. For instance, the energy purchase price may vary according to the hour and the month while the selling price of renewable energies is often constant. In this case, the energy cost is a piecewise linear function of the power consumption or production according to the hour, date, and direction of the power flow. On the other hand, the delivery cost of electricity is a non-linear function that is not investigated in this paper but constitutes a challenging prospect.

The power from the grid $\boldsymbol{P}_{\text {grid }}$ is decomposed in two parts, the power received $\boldsymbol{P}_{\text {grid }}^{+}$and the power sent $\boldsymbol{P}_{\text {grid }}^{-}$assuming that at least one of them is equal to zero:

$$
P_{\text {grid }, \mathrm{i}}=P_{\text {grid, } \mathrm{i}}^{+}+P_{\text {grid }, \mathrm{i}}^{-} \quad \forall i \in \llbracket 1, n_{\mathrm{t}} \rrbracket
$$

This allows changing the non-linear energy cost function by a piecewise linear function:

$$
\operatorname{cost}\left(P_{\text {grid,i }}\right)=\Delta t\left[C_{\text {grid, } \mathrm{i}}^{+} P_{\text {grid }, \mathrm{i}}^{+}+C_{\text {grid,i }}^{-} P_{\text {grid }, \mathrm{i}}^{-}\right] \quad \forall i \in \llbracket 1, n_{\mathrm{t}} \rrbracket
$$

where $\boldsymbol{C}_{\text {grid }}^{+}$and $\boldsymbol{C}_{\text {grid }}^{-}$are the costs for purchasing and selling electricity, respectively. They are changing according to the hour of the day and the day of the year.

Moreover, the investment cost is assumed to be a linear function:

$$
C_{\mathrm{inv}}\left(S_{\mathrm{PV}}, S_{\mathrm{W}}, P_{\mathrm{max}}, E_{\mathrm{max}}\right)=C_{\mathrm{sto}}^{\mathrm{E}} E_{\mathrm{max}}+C_{\mathrm{sto}}^{\mathrm{P}} P_{\mathrm{max}}+C_{\mathrm{PV}} S_{\mathrm{PV}}+C_{\mathrm{W}} S_{\mathrm{W}}
$$

As a new degree of freedom is introduced, $\boldsymbol{P}_{\text {grid }}^{+}$and $\boldsymbol{P}_{\text {grid }}^{-}$cannot be deduced from other power flows as before and the power balance expressed in (1)-(3) has to be reintroduced for the power and energy formulations, respectively as:

$$
\begin{aligned}
& P_{\text {load }, \mathrm{i}}-P_{\text {sto }, \mathrm{i}}-S_{\mathrm{PV}} P_{\mathrm{irr}, \mathrm{i}}-k_{\mathrm{p}} S_{\mathrm{W}} v_{\mathrm{adj}, \mathrm{i}}^{3}-P_{\text {grid }, \mathrm{i}}^{+}-P_{\text {grid }, \mathrm{i}}^{-}=0 \quad \forall i \in \llbracket 1, n_{\mathrm{t}} \rrbracket \\
& P_{\text {load }, \mathrm{i}}-\left[E_{\mathrm{sto}, \mathrm{i}-1}-E_{\mathrm{sto}, \mathrm{i}}\right] / \Delta t-S_{\mathrm{PV}} P_{\mathrm{irr}, \mathrm{i}}-k_{\mathrm{p}} S_{\mathrm{W}} v_{\mathrm{adj}, \mathrm{i}}^{3}-P_{\text {grid }, \mathrm{i}}^{+}-P_{\text {grid, } \mathrm{i}}^{-}=0 \quad \forall i \in \llbracket 1, n_{\mathrm{t}} \rrbracket
\end{aligned}
$$
stated as:

Therefore, the linear optimization problems with the power and energy formulations are respectively

$$
\begin{aligned}
& \left.\min _{S_{\mathrm{PV}}, S_{\mathrm{W}}, P_{\mathrm{max}}, E_{\mathrm{max}}, E_{\mathrm{ini}}, \boldsymbol{P}_{\mathrm{sto}}, P_{\text {grid }}^{+}, P_{\text {grid }}^{-}}\left\{\begin{array}{c}
C_{\mathrm{sto}}^{\mathrm{E}} E_{\mathrm{max}}+C_{\mathrm{sto}}^{\mathrm{P}} P_{\mathrm{max}}+C_{\mathrm{PV}} S_{\mathrm{PV}}+C_{\mathrm{W}} S_{\mathrm{W}} \\
+n_{\mathrm{y}} \Delta t \sum_{\mathrm{i}=1}^{\mathrm{n}_{\mathrm{t}}}\left(C_{\mathrm{grid}, \mathrm{i}}^{+} P_{\mathrm{grid}, \mathrm{i}}^{+}+C_{\mathrm{grid}, \mathrm{i}}^{-} P_{\mathrm{grid}, \mathrm{i}}^{-}\right.
\end{array}\right)\right\} \\
& \text {s.t. (4d), (10), (11), (20) } \\
& S_{\mathrm{PV}} \in R^{+}, S_{\mathrm{W}} \in R^{+}, P_{\mathrm{max}} \in R^{+}, E_{\max } \in R^{+}, E_{\mathrm{ini}} \in R^{+} \\
& \boldsymbol{P}_{\text {sto }} \in R^{\mathrm{n}_{\mathrm{t}}}, \boldsymbol{P}_{\text {grid }}^{+} \in R^{+\mathrm{n}_{\mathrm{t}}}, \boldsymbol{P}_{\text {grid }}^{-} \in R^{-\mathrm{n}_{\mathrm{t}}} \\
& \min _{S_{\mathrm{PV}}, S_{\mathrm{W}}, P_{\mathrm{max}}, E_{\mathrm{max}}, E_{\mathrm{ini}}, \boldsymbol{E}_{\mathrm{sto}}, P_{\text {grid }}^{+}, P_{\text {grid }}^{-}}\left\{\begin{array}{c}
C_{\mathrm{sto}}^{\mathrm{E}} E_{\mathrm{max}}+C_{\mathrm{sto}}^{\mathrm{P}} P_{\mathrm{max}}+C_{\mathrm{PV}} S_{\mathrm{PV}}+C_{\mathrm{W}} S_{\mathrm{W}} \\
+n_{\mathrm{y}} \Delta t \sum_{\mathrm{i}=1}^{\mathrm{n}_{\mathrm{t}}}\left(C_{\mathrm{grid}, \mathrm{i}}^{+} P_{\text {grid }, \mathrm{i}}^{+}+C_{\text {grid,i }}^{-} P_{\text {grid,i }}^{-}\right)
\end{array}\right\} \\
& \text {s.t. (5d), (6d), (14), (21) } \\
& S_{\mathrm{PV}} \in R^{+}, S_{\mathrm{W}} \in R^{+}, P_{\max } \in R^{+}, E_{\mathrm{max}} \in R^{+}, E_{\mathrm{ini}} \in R^{+} \\
& \boldsymbol{E}_{\text {sto }} \in R^{+\mathrm{n}_{\mathrm{t}}}, \boldsymbol{P}_{\text {grid }}^{+} \in R^{+\mathrm{n}_{\mathrm{t}}}, \boldsymbol{P}_{\text {grid }}^{-} \in R^{-\mathrm{n}_{\mathrm{t}}}
\end{aligned}
$$


The linearized optimization problems for both formulations include $3 n_{\mathrm{t}}+5=157685$ decision variables, one linear objective function, $n_{\mathrm{t}}+1=52561$ linear equality constraints, and $4 n_{\mathrm{t}}=210240$ linear inequality constraints. Table 1 summarizes the decision variables and constraints for both formulations.

Table 1. Description of both formulations for linearized optimization problem

\begin{tabular}{|c|c|c|c|}
\hline Formulation & & power & energy \\
\hline \multirow{2}{*}{ Variables } & common & \multicolumn{2}{|c|}{$S_{\mathrm{PV}}, S_{\mathrm{W}}, E_{\mathrm{max}}, P_{\mathrm{max}}, E_{\mathrm{ini}}, \boldsymbol{P}_{\mathrm{grid}}^{+}, \boldsymbol{P}_{\mathrm{grid}}^{-}$} \\
\hline & specific & $P_{\text {sto }}$ & $E_{\text {sto }}$ \\
\hline \multirow{3}{*}{ Constraints } & $\begin{array}{l}\text { energy } \\
\text { bounds }\end{array}$ & $0 \leq E_{\text {ini }}-\Delta t \sum_{\mathrm{j}=1}^{\mathrm{i}} P_{\text {sto }, \mathrm{j}} \leq E_{\max }$ & $0 \leq E_{\text {sto }, \mathrm{i}} \leq E_{\max }$ \\
\hline & $\begin{array}{l}\text { power } \\
\text { bounds }\end{array}$ & $-P_{\max } \leq P_{\text {sto }, \mathrm{i}} \leq P_{\max }$ & $-P_{\max } \Delta t \leq E_{\text {sto }, \mathrm{i}-1}-E_{\text {sto }, \mathrm{i}} \leq P_{\max } \Delta t$ \\
\hline & periodicity & $\sum_{\mathrm{i}=1}^{\mathrm{int}} P_{\text {sto }, \mathrm{i}}=0$ & $E_{\text {sto }, \mathrm{n}_{\mathrm{t}}}=E_{\mathrm{ini}}$ \\
\hline
\end{tabular}

Linear programming is used to solve the optimization problem for both formulations. In Matlab programming, specific matrices are required to express the equality and inequality constraints. Due to a time step of 10 minutes, each temporal variable induces 52560 unknowns for one year cycle thus sparse matrices are employed to reduce the memory requirements. In Table 2, both formulations are compared according to the size of matrices required to express the equality and inequality constraints such as the amount of memory required to store them by using full or sparse matrices.

Table 2. Comparison of memory requirements for both formulations $\left(n_{t}=52560\right)$

\begin{tabular}{ccccc}
\hline \hline \multirow{2}{*}{ Formulation } & \multicolumn{2}{c}{ power } & \multicolumn{2}{c}{ energy } \\
\cline { 2 - 5 } & Expression & Memory & Expression & Memory \\
\hline Size of matrices & $\left(5 n_{\mathrm{t}}+1\right)\left(3 n_{\mathrm{t}}+5\right)$ & $309 \mathrm{~GB}($ full $)$ & $\left(4 n_{\mathrm{t}}+1\right)\left(3 n_{\mathrm{t}}+5\right)$ & $247 \mathrm{~GB}$ (full) \\
Number of nonzero & $14 n_{\mathrm{t}}+n_{\mathrm{t}}^{2}$ & $62 \mathrm{~GB}($ sparse) & $14 n_{\mathrm{t}}+4$ & $17 \mathrm{MB}(\mathrm{sparse})$ \\
\hline \hline
\end{tabular}

Fig. 2.a. and Fig. 2.b. present respectively the nonzero elements of equality and inequality matrices for power and energy formulations during one day, i.e. 144 time steps. On Fig. 2.a., it can be seen that the power formulation induces a higher number of nonzero elements which makes difficult the storage of matrices for a high number of decision variables. By employing energy formulation, the memory required is greatly reduced and the optimization of HRPS is achievable in about one minute on a laptop computer for a complete year. The implementation is made with Matlab 2015b and the dual-simplex algorithm in linprog function from the Optimization toolbox. The tolerance has to be reduced to $10^{-9}$ to achieve a good precision for a complete year. Other options take the default values.
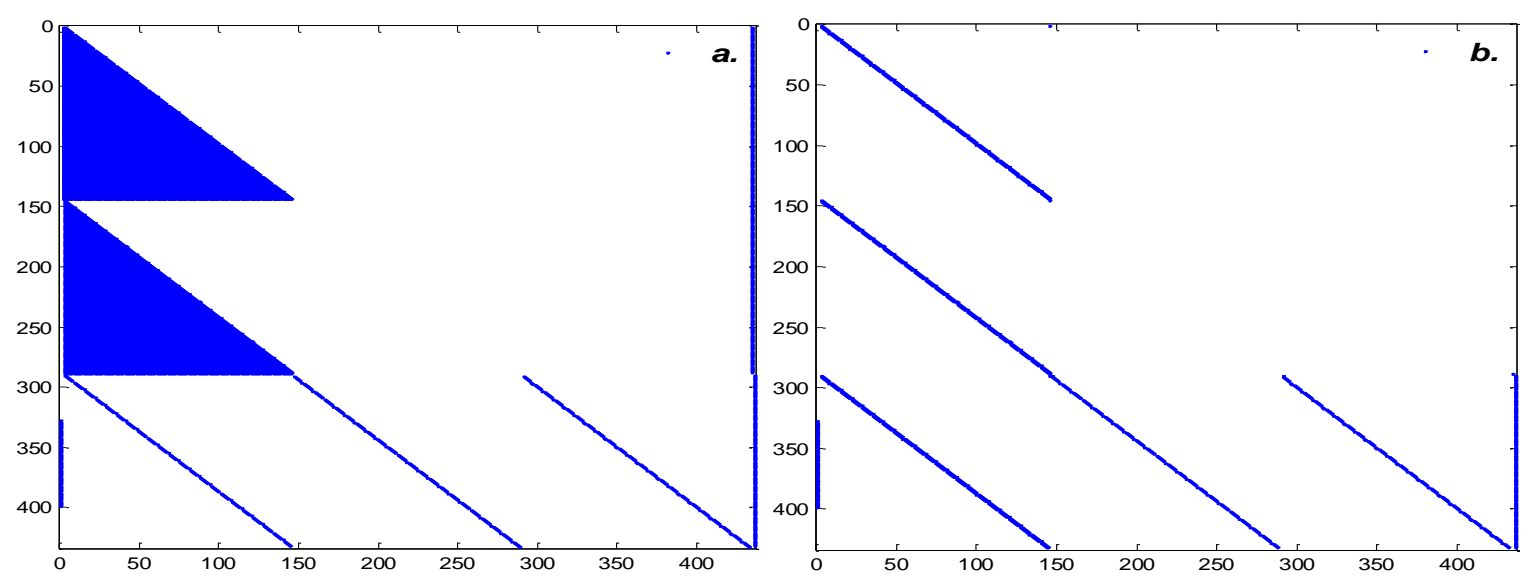

Figure 2. Comparison of matrices for one day. Each column is a variable and each row is a constraint.

The linearization of costs appears to be a good way to get an approximate solution of the HRPS optimization problem but the prospect is to optimize with the real costs that are non-linear functions. Therefore, new strategies are required for the non-linear optimization with a high number of decision variables. 


\section{COLLABORATIVE OPTIMIZATION}

The principle of collaborative optimization is to decompose the complete problem into sub-problems, each of them having a lower number of decision variables [6]. Sequential quadratic programming (SQP) and active-set, among others, are algorithms able to accurately solve non-linear optimization problems with numerous active constraints at optimum in a reduced number of evaluations. Unfortunately, the number of variables is limited to hundreds. Interior-point method (IPM) can extend the number of variables to thousands but the precision of the solution may decrease because of the barrier method used to handle constraints. Attempts to solve problems (13) and (16) by using SQP and IPM have been carried out with an increasing number of time steps (Fig. 3). It appears that the convergence of SQP is not achieved in an affordable computing time beyond 432 time steps ( 3 days) while the limit of IPM is 1296 time steps (9 days). For an increasing number of time steps, IPM shows better solution and lower computing time compared to SQP. However, only the solution found by SQP for a time range of one day is as good as LP.
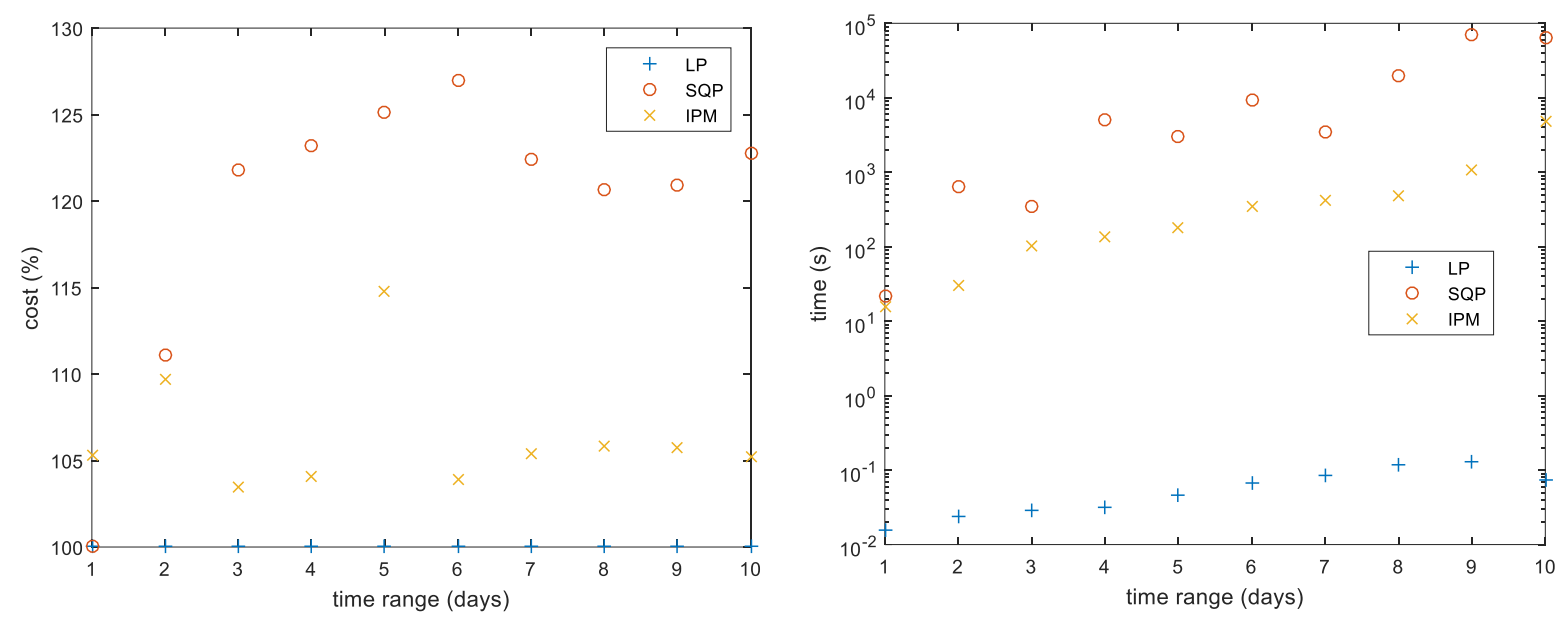

Figure 3. Comparison of algorithms to solve HRPS complete problem: cost (left) and time (right). The cost is normalized with respect to LP value.

Therefore, the decomposition of the HRPS problem is guided by the number of variables in each subproblem. It is proposed to decompose in two levels: year and day. This is shown in Fig. 4 where the year level is at the top and the day level at bottom. For the sake of simplicity, the year is reduced to 7 days in this figure. At the year level, the design variables are the four dimensional parameters since their values are not time-dependent. At the day level, the idea is to decompose the complete year in $n_{\mathrm{d}}=365$ days with optimizations made in parallel. This is enabled by introducing additional linking variables at the year level that are the amount of energy in the storage system at the beginning of each day. Additional constraints are added at the day level to impose the amount of energy stored at the beginning and the end of each day. The number of design variables for one day is $n_{\mathrm{t}} / n_{\mathrm{d}}=$ 144 , that is also the number of time steps for this period, i.e. 24 hours multiplied by 6 time steps per hour. At the year level, the number of design variables is $n_{\mathrm{d}}+4=369$ corresponding to $n_{\mathrm{d}}=365$ linking variables and 4 dimensional parameters.

The minimum energy costs (24) at the day level given the values of the dimensional and linking variables are found by $n_{\mathrm{d}}$ optimizations in parallel and added as a second member in the objective function (25) at the year level.

$$
\begin{aligned}
& \underset{E_{\text {sto }}}{C_{\text {ener, }}}=\min \sum_{i=1+(\mathrm{d}-1) \mathrm{n}_{\mathrm{t}} / \mathrm{n}_{\mathrm{d}}}^{\mathrm{d} \mathrm{n}_{\mathrm{t}} / \mathrm{n}_{\mathrm{d}}} \cos t\left(P_{\mathrm{load}, \mathrm{i}}-P_{\mathrm{PV}, \mathrm{i}}-P_{\mathrm{W}, \mathrm{i}}-\left[E_{\text {sto,i-1 }}-E_{\text {sto }, \mathrm{i}}\right] / \Delta t\right)
\end{aligned}
$$

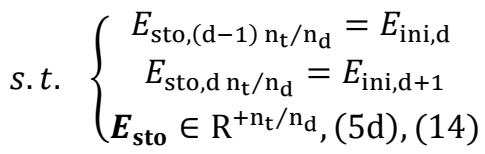

$$
\mathrm{OF}\left(S_{\mathrm{PV}}, S_{\mathrm{W}}, P_{\mathrm{max}}, E_{\mathrm{max}}, \boldsymbol{E}_{\mathrm{ini}}\right)=C_{\mathrm{inv}}\left(S_{\mathrm{PV}}, S_{\mathrm{W}}, P_{\mathrm{max}}, E_{\mathrm{max}}\right)+n_{\mathrm{y}} \sum_{\mathrm{d}=1}^{\mathrm{n}_{\mathrm{d}}} C_{\mathrm{ener}, \mathrm{d}}
$$




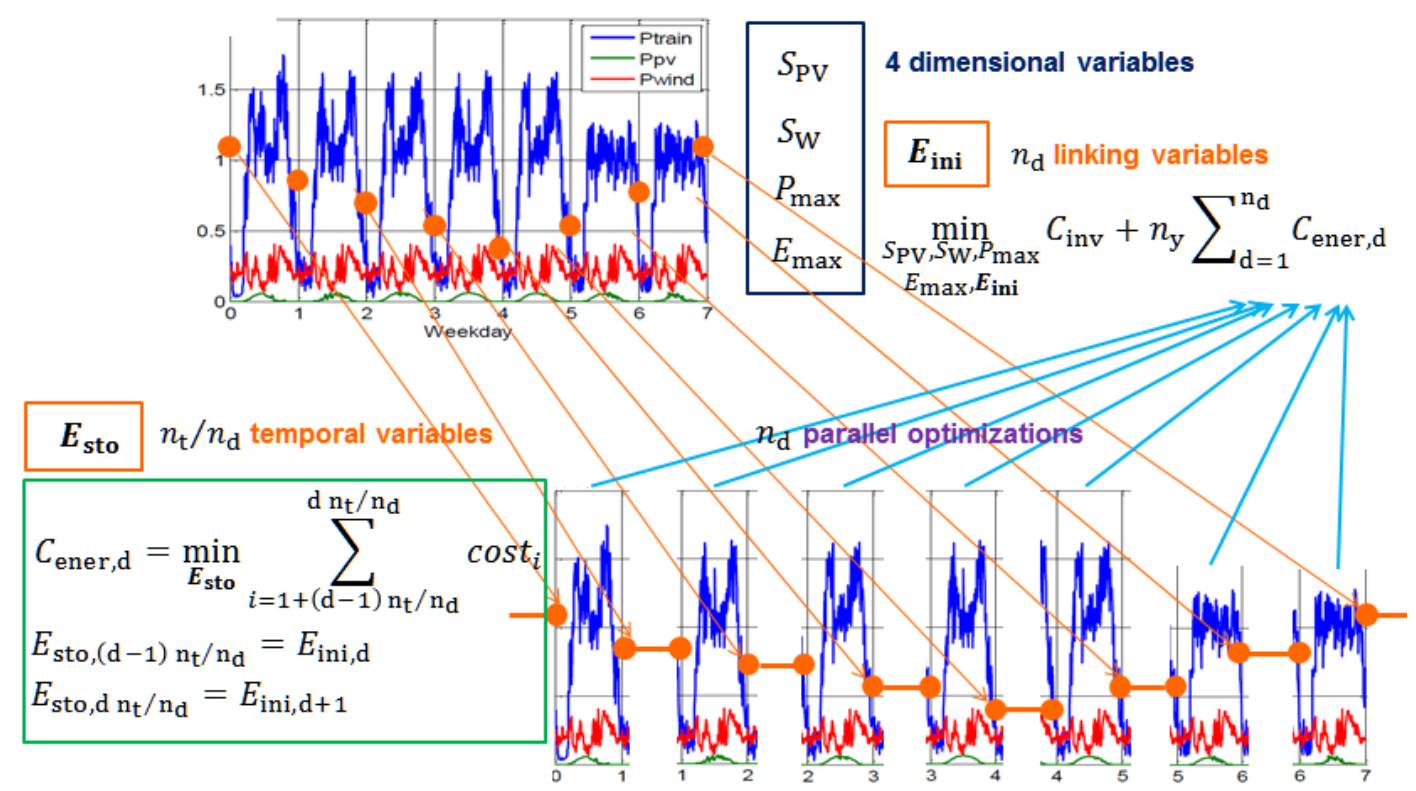

Figure 4. Decomposition of the HRPS in two levels.

As the objective function at the year level includes the results of optimizations at day level, those lasts are nested in the year level model as shown in Fig. 5. This leads to a double-loop approach with high computing time and slow convergence. Two cases are considered for the inner-loop. In the first case (CO 1st case), the energy cost is a non-linear function and problems (24) are solved with non-linear programming (NLP) and distributed computing. In the second case (CO 2nd case), the energy cost is approximated by a piecewise linear function and problems (24) are solved with linear programming. For both cases, the outer-loop that minimizes (25) requires NLP because the energy cost for all days $C_{\text {ener,d }}$ are implicit functions. The derivatives of $C_{\text {ener,d }}$ relative to the variables $S_{\mathrm{PV}}, S_{\mathrm{W}}, P_{\mathrm{max}}, E_{\mathrm{max}}, \boldsymbol{E}_{\mathrm{ini}}$ are computed by using the finite difference technique. There is no guaranty of convergence for the outer-loop due to the fact that $C_{\text {ener,d }}$ may not be convex. However, all the constraints are linear and the feasible space is thus convex. This double-loop approach appears to have unaffordable computing time even for a small time range in the first case while the same solution as LP is found in a reasonable computing time for few days in the second case (Fig. 6). The collaborative optimization with linear programming in the innerloop $\left(2^{\text {nd }}\right.$ case $)$ will be investigated for a complete year in the results part.

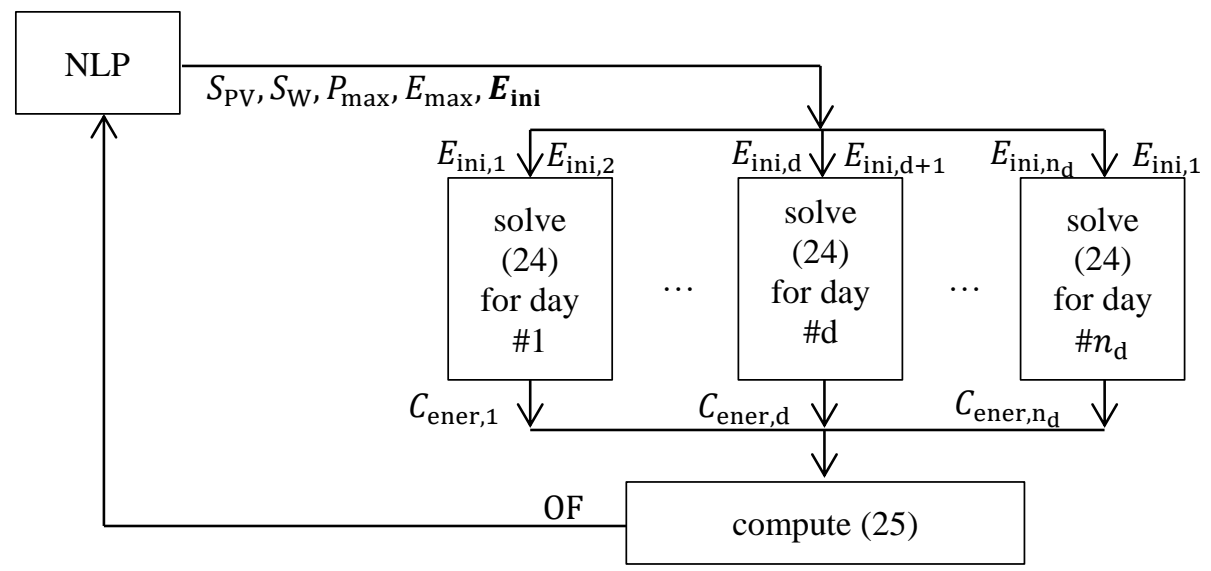

Figure 5. Collaborative optimization (double-loop) structure. 

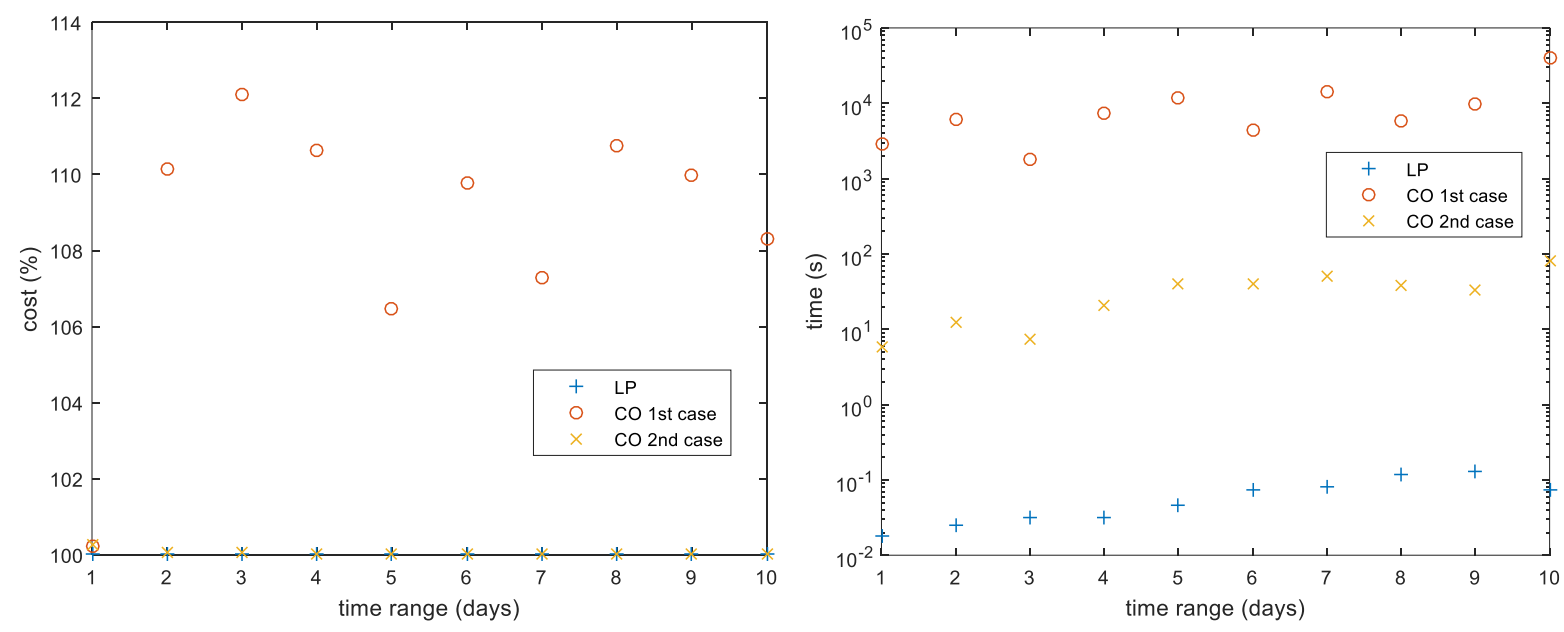

Figure 6. Comparison of algorithms to solve HRPS problem with double-loop approaches: cost (left) and time (right). The cost is normalized with respect to LP value.

\section{MULTILEVEL OPTIMIZATION}

The advantage of multilevel optimization is to avoid the nested optimization by using an approach where the optimizations are solved sequentially. At the year level, the optimization is performed by using substitute models $C_{\text {ener,d }}^{\sim}$ of the result of optimizations at the day level $C_{\text {ener,d }}$ for each day. This way, the costly optimizations at the day level are replaced by fast evaluations of functions at the year level that are usually linear. Then, the optimizations are made at the day level and the substitute models are refined. The loop between levels stops when the discrepancies between the substitute models and the minimum energy costs are negligible. Figure 7 gives the flowchart of this approach and the main steps are detailed below.

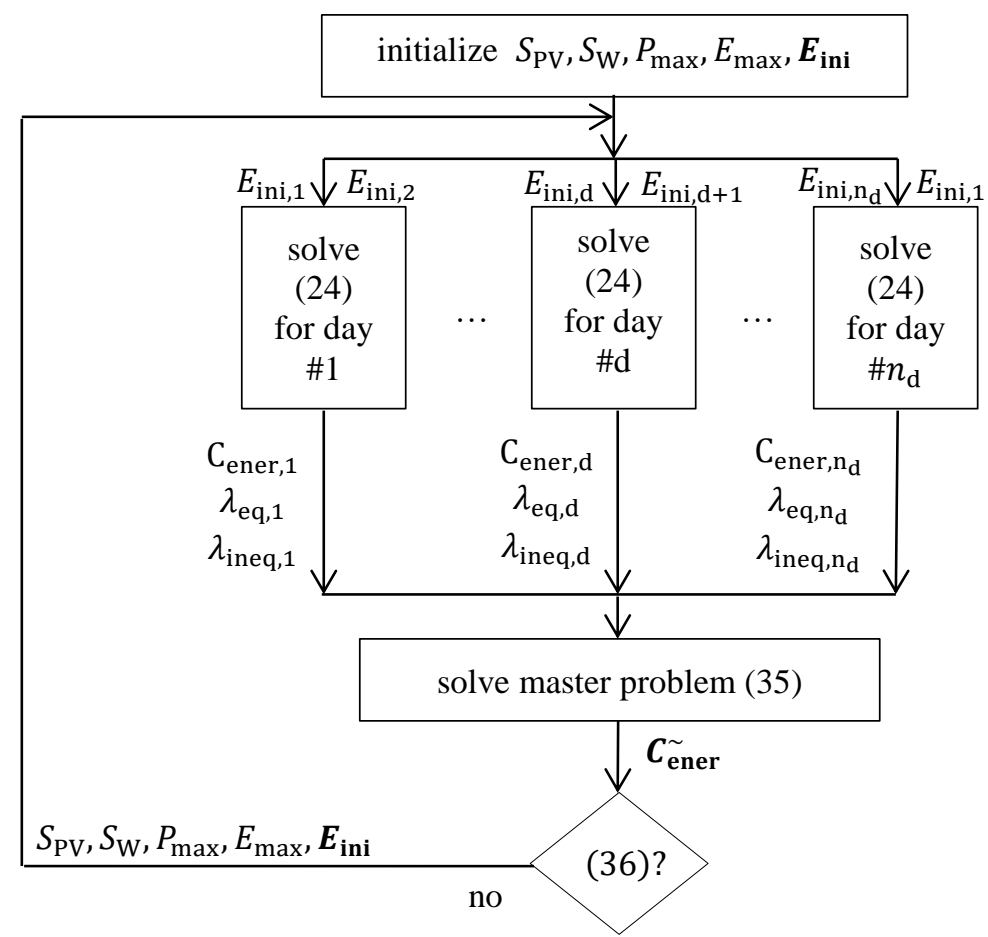

Figure 7. Multilevel optimization (sequential) structure.

As the objective function $C_{\text {ener,d }}=f\left(E_{\text {sto, } 1+(\mathrm{d}-1) \mathrm{n}_{\mathrm{t}} / \mathrm{n}_{\mathrm{d}}}, \cdots, E_{\text {sto, } \mathrm{d}} / \mathrm{n}_{\mathrm{d}}\right)$ expressed in (24) is an implicit function of the design variables at the day level, the difficulty is to build a substitute model $\tilde{C}_{\text {ener,d }}=$ $s_{d}\left(E_{\mathrm{max}}, P_{\mathrm{max}}, S_{\mathrm{PV}}, S_{\mathrm{W}}, E_{\mathrm{ini}, \mathrm{d}}, E_{\mathrm{ini}, \mathrm{d}+1}\right)$ that is an explicit function of the design variables at the year level.

Based on the theory of duality, the Benders decomposition [7], [8] takes advantage of the information on the Lagrange multipliers to define a lower bound for the objective function of the sub-problems, as detailed in Appendix. When the objective function and the constraints of the sub-problems are linear, and the objective 
function and the constraints of the master problem (at the year level) are continuous, then Benders decomposition converges in a finite number of steps [8]. If the whole problem has a finite optimum solution, Benders decomposition converges to an optimum solution. Hence, the application of Benders decomposition on problem (22) or (23) gives an optimal solution. If the objective function or the constraints of the sub-problems are no longer linear, the problem can be solved using a generalized Benders decomposition [9]. This method is proven to be convergent when the sub-problems consist in minimizing a convex function over a convex constraint set [9].

In the HRPS optimization problem, the objective functions are the costs of energy for all days. In the subproblems, the decision variables $E_{\mathrm{max}}, P_{\mathrm{max}}, S_{\mathrm{PV}}, S_{\mathrm{W}}, \boldsymbol{E}_{\text {ini }}$ at the year level are constant. The constraints $(5 \mathrm{~d}),(6 \mathrm{~d})$, (14), and (15) are rewritten such as these variables appear in their RHS:

$$
\begin{aligned}
& E_{\text {sto,i }} \leq E_{\max } \quad \forall i \in \llbracket 1+(d-1) n_{\mathrm{t}} / n_{\mathrm{d}}, d n_{\mathrm{t}} / n_{\mathrm{d}} \rrbracket \\
& -E_{\mathrm{sto}, 1+(\mathrm{d}-1) \mathrm{n}_{\mathrm{t}} / \mathrm{n}_{\mathrm{d}}} \leq P_{\max } \Delta t-E_{\mathrm{ini}, \mathrm{d}} \\
& E_{\mathrm{sto}, \mathrm{i}-1}-E_{\mathrm{sto}, \mathrm{i}} \leq P_{\max } \Delta t \quad \forall i \in \llbracket 2+(d-1) n_{\mathrm{t}} / n_{\mathrm{d}}, d n_{\mathrm{t}} / n_{\mathrm{d}} \rrbracket \\
& E_{\text {sto }, 1+(\mathrm{d}-1) \mathrm{n}_{\mathrm{t}} / \mathrm{n}_{\mathrm{d}}} \leq P_{\max } \Delta t+E_{\text {ini,d }} \\
& E_{\text {sto }, \mathrm{i}}-E_{\text {sto }, \mathrm{i}-1} \leq P_{\max } \Delta t \quad \forall i \in \llbracket 2+(d-1) n_{\mathrm{t}} / n_{\mathrm{d}}, d n_{\mathrm{t}} / n_{\mathrm{d}} \rrbracket \\
& -E_{\text {sto }, 1+(\mathrm{d}-1) \mathrm{n}_{\mathrm{t}} / \mathrm{n}_{\mathrm{d}}}+P_{\text {grid }, 1+(\mathrm{d}-1) \mathrm{n}_{\mathrm{t}} / \mathrm{n}_{\mathrm{d}}} \Delta t=P_{\text {load }, 1+(\mathrm{d}-1) \mathrm{n}_{\mathrm{t}} / \mathrm{n}_{\mathrm{d}}} \Delta t-S_{\mathrm{PV}} P_{\text {irr }, 1+(\mathrm{d}-1) \mathrm{n}_{\mathrm{t}} / \mathrm{n}_{\mathrm{d}}} \Delta t \\
& -k_{\mathrm{p}} S_{\mathrm{W}} v_{\mathrm{adj}, 1+(\mathrm{d}-1) \mathrm{n}_{\mathrm{t}} / \mathrm{n}_{\mathrm{d}}}^{3} \Delta t-E_{\mathrm{ini}, \mathrm{d}} \\
& E_{\text {sto }, \mathrm{i}-1}-E_{\text {sto }, \mathrm{i}}+P_{\text {grid,i }} \Delta t=\left[P_{\text {load }, \mathrm{i}}-S_{\mathrm{PV}} P_{\mathrm{irr}, \mathrm{i}}-k_{\mathrm{p}} S_{\mathrm{W}} v_{\mathrm{adj}, \mathrm{i}}^{3}\right] \Delta t \\
& \forall i \in \llbracket 2+(d-1) n_{\mathrm{t}} / n_{\mathrm{d}}, d n_{\mathrm{t}} / n_{\mathrm{d}} \rrbracket \\
& E_{\text {sto }, \mathrm{n}_{\mathrm{t}} / \mathrm{n}_{\mathrm{d}}}=E_{\mathrm{ini}, \mathrm{d}+1}
\end{aligned}
$$

The algorithm starts with an initial value of the variables at the year level. After solving the sub-problems, the Lagrange multipliers are available for all constraints: $\left(\lambda_{\text {ineq } 1, i}\right)_{1+(d-1) n_{t} / n_{d} \leq i \leq d n_{t} / n_{d}}$ for the inequality constraint (26), $\lambda_{\text {ineq2,1+(d-1) } n_{t} / n_{d}}$ for (27), $\left(\lambda_{\text {ineq2,i }}\right)_{2+(d-1) n_{t} / n_{d} \leq i \leq d n_{t} / n_{d}}$ for $(28), \lambda_{\text {ineq3,1+(d-1) } n_{t} / n_{d}}$ for (29), $\quad\left(\lambda_{\text {ineq } 3, i}\right)_{2+(d-1) n_{t} / n_{d} \leq i \leq d n_{t} / n_{d}}$ for (30), $\quad \lambda_{\text {eq } 1,1+(d-1) n_{t} / n_{d}}$ for the equality constraint (31), $\left(\lambda_{\text {eq1 }, i}\right)_{2+(d-1) n_{t} / n_{d} \leq i \leq d n_{t} / n_{d}}$ for (32), and $\lambda_{\text {eq2 }}$ for (33). The multiplier is a measurement of the expected decrease of the energy cost for an increase of the RHS of a constraint in the sub-problem. As all RHS are expressed explicitly as linear functions of the variables at the year level, the lower bounds for all energy costs $\widetilde{\boldsymbol{C}}_{\text {ener }}$ are deduced and introduced as additional constraints (34) in the optimization problem at the year level as explained in the Appendix.

$$
\begin{aligned}
& -\tilde{C}_{\text {ener }, \mathrm{d}}-\sum_{\mathrm{i}=1+(\mathrm{d}-1) \mathrm{n}_{\mathrm{t}} / \mathrm{n}_{\mathrm{d}}}^{\mathrm{d} \mathrm{n}_{\mathrm{t}} / \mathrm{n}_{\mathrm{d}}} \lambda_{\text {ineq } 1, \mathrm{i}} E_{\max }-\sum_{\mathrm{i}=1+(\mathrm{d}-1) \mathrm{n}_{\mathrm{t}} / \mathrm{n}_{\mathrm{d}}}^{\mathrm{d} \mathrm{n}_{\mathrm{t}} / \mathrm{n}_{\mathrm{d}}}\left(\lambda_{\text {ineq } 2, \mathrm{i}}+\lambda_{\text {ineq } 3, \mathrm{i}}\right) P_{\max } \Delta t+ \\
& \left(\lambda_{\text {ineq2,1+(d-1) } \mathrm{n}_{\mathrm{t}} / \mathrm{n}_{\mathrm{d}}}-\lambda_{\text {ineq } 3,1+(\mathrm{d}-1) \mathrm{n}_{\mathrm{t}} / \mathrm{n}_{\mathrm{d}}}+\lambda_{\text {eq } 1,1+(\mathrm{d}-1) \mathrm{n}_{\mathrm{t}} / \mathrm{n}_{\mathrm{d}}}\right) E_{\text {ini,d }}-\lambda_{\text {eq2 }} E_{\mathrm{ini}, \mathrm{d}+1} \\
& +\Delta t\left[\lambda_{\mathrm{eq} 1,1+(\mathrm{d}-1) \mathrm{n}_{\mathrm{t}} / \mathrm{n}_{\mathrm{d}}} \cdots \lambda_{\mathrm{eq} 1, \mathrm{~d} \mathrm{n} \mathrm{t} / \mathrm{n}_{\mathrm{d}}}\right]\left\{\left[\begin{array}{c}
P_{\mathrm{irr}, 1+(\mathrm{d}-1) \mathrm{n}_{\mathrm{t}} / \mathrm{n}_{\mathrm{d}}} \\
\vdots \\
P_{\mathrm{irr}, \mathrm{d} \mathrm{n} \mathrm{t}} / \mathrm{n}_{\mathrm{d}}
\end{array}\right] S_{\mathrm{PV}}+k_{\mathrm{p}}\left[\begin{array}{c}
v_{\mathrm{adj}, 1+(\mathrm{d}-1) \mathrm{n}_{\mathrm{t}} / \mathrm{n}_{\mathrm{d}}}^{3} \\
\vdots \\
v_{\mathrm{adj}, \mathrm{d} \mathrm{n}_{\mathrm{t}} / \mathrm{n}_{\mathrm{d}}}^{3}
\end{array}\right] S_{\mathrm{W}}\right\} \\
& \leq\left[\begin{array}{lll}
\lambda_{\text {eq } 1,1+(\mathrm{d}-1) \mathrm{n}_{\mathrm{t}} / \mathrm{n}_{\mathrm{d}}} & \cdots & \lambda_{\text {eq } 1, \mathrm{~d} \mathrm{n}} / \mathrm{n}_{\mathrm{d}}
\end{array}\right]\left[\begin{array}{c}
P_{\text {load }, 1+(\mathrm{d}-1) \mathrm{n}_{\mathrm{t}} / \mathrm{n}_{\mathrm{d}}} \\
\vdots \\
P_{\text {load }, \mathrm{d} \mathrm{n}_{\mathrm{t}} / \mathrm{n}_{\mathrm{d}}}
\end{array}\right] \Delta t
\end{aligned}
$$

The master problem (35) at the year level is then solved with these additional constraints, one for each day, and new values for the design variables are deduced such as the values of the expected energy costs for all days, $\tilde{C}_{\text {ener,d }}$. At each iteration, $n_{\mathrm{d}}$ new constraints on the energy costs are added. 


$$
\begin{aligned}
& \min _{S_{\mathrm{PV}}, S_{\mathrm{W}}, P_{\mathrm{max}}, E_{\mathrm{max}}, E_{\mathrm{ini}}, \widetilde{C}_{\mathrm{ener}}} C_{\mathrm{inv}}\left(S_{\mathrm{PV}}, S_{\mathrm{W}}, P_{\mathrm{max}}, E_{\mathrm{max}}\right)+n_{\mathrm{y}} \sum_{\mathrm{d}=1}^{\mathrm{n}_{\mathrm{d}}} C_{\mathrm{ener}, \mathrm{d}}^{\sim} \\
& \text { S.t. }(34) \forall d \in \llbracket 1, n_{\mathrm{d}} \rrbracket \\
& S_{\mathrm{PV}} \in R^{+}, S_{\mathrm{W}} \in R^{+}, P_{\max } \in R^{+}, E_{\max } \in R^{+}, \boldsymbol{E}_{\text {ini }} \in R^{+\mathrm{n}_{\mathrm{d}}}, \boldsymbol{C}_{\text {ener }}^{\sim} \in R^{\mathrm{n}_{\mathrm{d}}}
\end{aligned}
$$

The algorithm stops if the gap between the sums of energy costs found at both levels is small enough:

$$
\left|\sum_{\mathrm{d}=1}^{\mathrm{n}_{\mathrm{d}}} \tilde{C}_{\text {ener,d }}-\sum_{\mathrm{d}=1}^{\mathrm{n}_{\mathrm{d}}} C_{\text {ener,d }}\right| \leq \varepsilon
$$

where $\varepsilon$ is the precision chosen equal to $1 €$ what is about $10^{-7}$ relative to the minimum value of the objective function.

\section{RESULTS AND COMPARISON}

As already mentioned, the energy cost is assumed piecewise linear in order to compare the results of collaborative optimization and Benders decomposition to the solving of the complete optimization problem with linear programming. As expected, this last gives the lower total cost in the shortest time and is considered as the reference for comparison.

Figures 8 show the value of the objective function and the computing time for the three approaches when the number of days considered in the problem increases. LP is linear programming, $\mathrm{CO}$ is collaborative optimization with linear programming for the inner-loop, and BD is the Benders decomposition. The cost is normalized with respect to LP value. It can be seen that BD gives the same solution as LP with about 5 times more computing time but this ratio decreases as the number of days considered increases. CO fails to find the solution when considering more than 120 days. The time required by $\mathrm{CO}$ is about one thousand times more than LP.
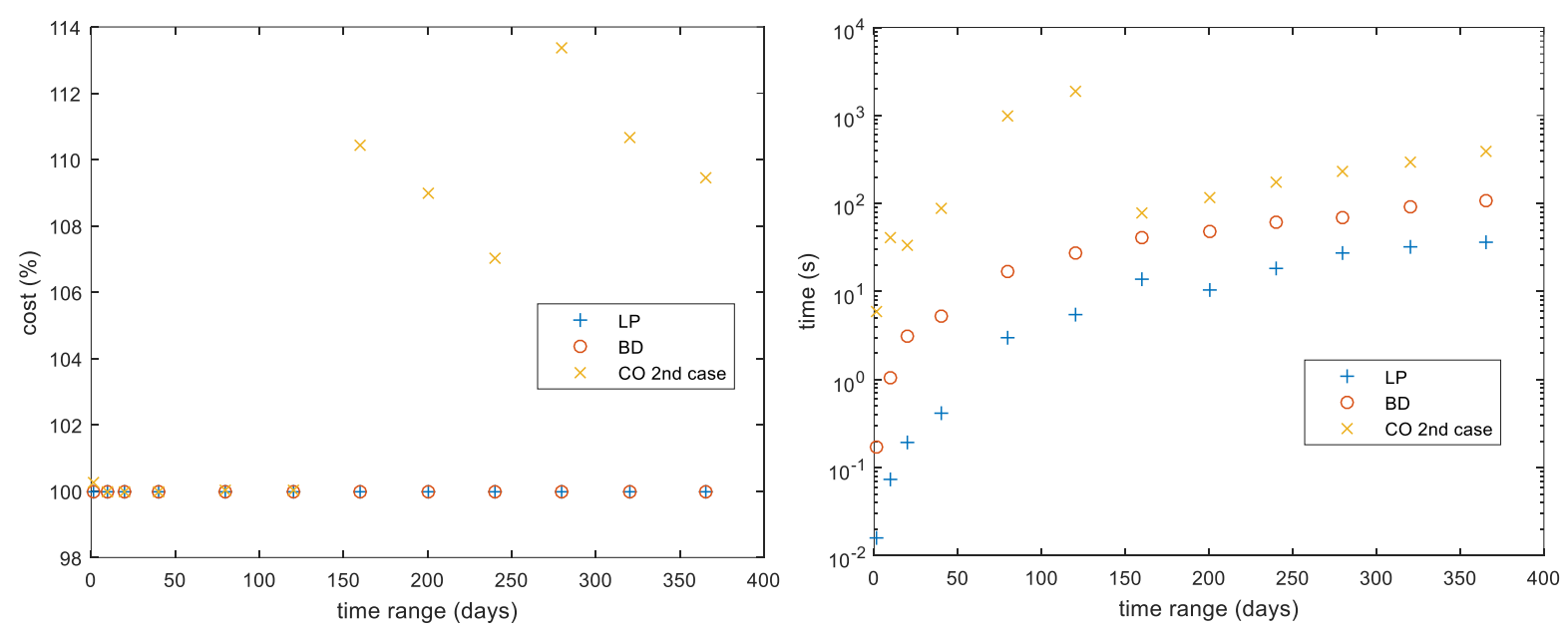

Figure 8. Comparison of approaches to solve HRPS problem: cost (left) and time (right).

\section{CONCLUSIONS}

Benders decomposition appears to be very efficient for the optimization of HRPS on a large time range taking into account the control of the storage system. For a piecewise linear cost function, the design is performed in a few minutes on a laptop computer for a complete year. The solution is the same as the one found with linear programming and the computing time is about 5 times higher only. Collaborative optimization fails to find the same solution and its computing time is at least one hundred times higher.

As the energy cost function is not linear in reality, the linear programming cannot be used. Therefore, one prospect is to apply Benders decomposition to design the HRPS with a non-linear energy cost function as it is not possible to solve this optimization problem as a whole.

\section{REFERENCES}

[1] M. Aeberhard, C. Courtois, and P. Ladoux, "Railway traction power supply from the state of the art to future trends", International Symposium on Power Electronics Electrical Drives Automation and Motion (SPEEDAM), pp. 1350-1355, 2010.

[2] C. Clastres, T. Ha Pham, F. Wurtz, and S. Bacha, "Ancillary services and optimal household energy management with photovoltaic production”, Energy, vol. 35, no. 1, pp. 55-64, 2010.

[3] R. Rigo-Mariani, B. Sareni, and X. Roboam, "Optimal sizing integrating power management for a microgrid with storage", Optimization and Inverse Problems in Electromagnetic, Delft, Holland, 2014.

[4] H.K. Fathy, J.A. Reyer, P.Y. Papalambros and A.G. Ulsoy, "On the coupling between the plant and controller optimization problems", Proceedings of the American Control Conference, Arlington, VA, June 25-27, 2001. 
[5] J. Aubry, H. Ben Ahmed, and B. Multon, "Sizing optimization methodology of a surface permanent magnet machine-converter system over a torque-speed operating profile : Application to wave energy converter", IEEE Trans. Industrial Electronics, vol. 59, no. 5, pp. 2116-2125, 2012.

[6] P.M. Zadeh, V.V. Toropov, and A.S. Wood. "Metamodel-based collaborative optimization framework', Structural and Multidisciplinary Optimization, vol. 38, no. 2, pp. 103-115, 2009.

[7] A.M. Costa, "A survey on Benders decomposition applied to fixed-charge network design problems", Computers \& Operations Research, vol. 32, no. 6, pp. 1429-1450, 2005.

[8] J.F. Benders, "Partitioning Procedures for Solving Mixed-Variables Programming Problems", Numerische Mathematik, vol. 4, no. 1, pp. 238-252, 1962.

[9] A.M. Geoffrion, "Generalized benders decomposition”, Journal of optimization theory and applications, vol. 10, no. 4, pp. 237-260, 1972 .

\section{APPENDIX: BENDERS DECOMPOSITION}

We consider a linear optimization problem with two sets of variables. The complete formulation is:

(A1) $\quad \min _{\mathrm{x}, \mathrm{y}} c^{\mathrm{t}} x+d^{\mathrm{t}} y$

$$
\begin{gathered}
\text { s.t. } \quad A_{\mathrm{eq}} x+B_{\text {eq }} y=b_{\mathrm{eq}} \\
D x \leq e
\end{gathered}
$$

(A3) with

$$
\begin{aligned}
& l b_{\mathrm{x}} \leq x \leq u b_{\mathrm{x}} \\
& l b_{\mathrm{y}} \leq y \leq u b_{\mathrm{y}}
\end{aligned}
$$

In order to perform the Benders decomposition, the variables $x$ are assumed to belong to the master problem while the variables $y$ are in the sub-problem. Classically, the variables in the master problem are integer and the ones in the sub-problem are continuous but Benders decomposition can also be applied to problems with only continuous variables.

At the beginning, an initial value $x_{0}$ for the variables in the master problem is given. The first iteration $(k=1)$ starts by solving the sub-problem expressed as:

$$
f_{\mathrm{k}}=\min _{\mathrm{y}} d^{\mathrm{t}} y
$$

$$
\begin{gathered}
B y \leq b-A x_{\mathrm{k}-1} \\
\text { s.t. } \\
B_{\mathrm{eq}} y=b_{\mathrm{eq}}-A_{\mathrm{eq}} x_{\mathrm{k}-1}
\end{gathered}
$$

(A6) with $\quad l b_{\mathrm{y}} \leq y \leq u b_{\mathrm{y}}$

After solving the sub-problem (A4)-(A6), a marginal cost or Lagrange multiplier is available for each constraint: $\lambda_{\text {ineq }}$ for inequality constraint, $\lambda_{\text {eq }}$ for equality constraint, $\lambda_{\mathrm{ub}}$ for upper bound, and $\lambda_{\mathrm{lb}}$ for lower bound. The multiplier is a measurement of the expected decrease of the objective for an increase of the RHS of the constraint. The multiplier for an inequality constraint is positive if the constraint is active else it is equal to zero. For equality constraint, the multiplier is positive or negative.

The range constraint (A6) is transformed in two inequality constraints to standardize the notation:

with

$$
\begin{gathered}
y \leq u b_{y} \\
-y \leq-l b_{y}
\end{gathered}
$$

The second step in the iteration is to solve the master problem expressed as:

$$
\left[x_{\mathrm{k}}, f_{\mathrm{k}}^{\sim}\right]=\underset{\mathrm{x}, \mathrm{f}^{\sim}}{\operatorname{argmin}} c^{\mathrm{t}} x+f^{\sim}
$$

$$
-f^{\sim} \leq\left[\begin{array}{llll}
\lambda_{\text {ineq }}^{\mathrm{t}} & \lambda_{\mathrm{eq}}^{\mathrm{t}} & \lambda_{\mathrm{ub}}^{\mathrm{t}} & \lambda_{\mathrm{lb}}^{\mathrm{t}}
\end{array}\right]\left[\begin{array}{c}
b-A x \\
b_{\mathrm{eq}}-A_{\mathrm{eq}} x \\
u b_{\mathrm{y}} \\
-l b_{\mathrm{y}}
\end{array}\right]
$$


(A10) with $l b_{\mathrm{x}} \leq x \leq u b_{\mathrm{x}}$

where $f^{\sim}$ is an additional design variable in the master problem and acts as a substitute of the second member of the objective function of the whole problem. This variable is attracted to $-\infty$ since it is added to the objective function but the constraint (A9) on $f^{\sim}$ prevents to reach it. This constraint comes from the duality theory.

The inequality constraints in (A9) are written in the standardized notation as:

(A11) $\quad$ s.t. $\quad-f^{\sim}+\left(\lambda_{\text {ineq }}^{\mathrm{t}} A+\lambda_{\mathrm{eq}}^{\mathrm{t}} A_{\mathrm{eq}}\right) x \leq \lambda_{\text {ineq }}^{\mathrm{t}} b+\lambda_{\mathrm{eq}}^{\mathrm{t}} b_{\mathrm{eq}}+\lambda_{\mathrm{ub}}^{\mathrm{t}} u b_{y}-\lambda_{\mathrm{lb}}^{\mathrm{t}} l b_{y}$

The iterations are continued until a stopping criterion is fulfilled. This stopping criterion is usually:

(A12) $\quad\left|f_{\mathrm{k}}-f_{\mathrm{k}}^{\sim}\right| \leq \varepsilon$

where $\varepsilon$ is the requested precision. A new constraint on $f^{\sim}$ is added to the set of inequality constraint at each iteration. 